\title{
A review of the anatomy and a step-by-step visual guide to performing an ultrasound-guided supraclavicular brachial plexus block
}

\author{
Govender $\mathrm{S}^{1,2}$ (D), Möhr $\mathrm{D}^{2,3}$, Tshabalala ZN² $\mathbb{D}$, Van Schoor $\mathrm{A}^{2^{*}}$ (iD \\ 'Department of Anatomy, School of Medicine, Sefako Makgatho Health Sciences University \\ 2 Department of Anatomy, Section of Clinical Anatomy, School of Medicine, Faculty of Health Sciences, University of Pretoria \\ ${ }^{3}$ Private Practice, Anaesthesiologist \\ ${ }^{*}$ Corresponding author, email: albert.vanschoor@up.ac.za
}

Background: The aim of this description is to provide step-by-step guidelines for performing an ultrasound-guided supraclavicular brachial plexus nerve block.

Methods: The brachial plexus in the supraclavicular fossa of sixty healthy volunteers was scanned in the horizontal/transverse plane. The relevant regional anatomy was studied to identify the muscular and vascular structures seen on the ultrasound screen.

Results: The entire process was documented and a standard, step-by-step guide to performing ultrasound-guided supraclavicular brachial plexus blocks was developed.

Conclusion: This description serves as a comprehensive guide to a technique for performing ultrasound-guided supraclavicular brachial plexus blocks safely and successfully. It also aims to provide the reader with the background knowledge of the technique and the surrounding regional anatomy.

Keywords: brachial plexus, nerve blocks, supraclavicular, ultrasound-guided, regional anatomy.

\section{Introduction}

The supraclavicular approach to the brachial plexus block was first described in the early $20^{\text {th }}$ century and is arguably the most preferred brachial plexus (BP) approach with the highest success rate. ${ }^{1}$ With this approach, the trunks/divisions of the $\mathrm{BP}$ are compact and superficial to the skin making it easy to visualise on ultrasound. ${ }^{1}$ Due to the limited surface area, the entire BP is anaesthetised. The supraclavicular block also has the most widespread extent of sensory blockade among all the BP approaches and is ideal for providing dense, rapid onset, and efficient anaesthesia and analgesia for procedures from the shoulder joint and mid-humerus proximally, to the hand distally. ${ }^{1}$ According to the literature, the supraclavicular approach proves to be one of the safest and most effective techniques. ${ }^{2}$

Alternative approaches include the interscalene, infraclavicular and axillary BP nerve blocks. The interscalene BP block, which is indicated for surgery of the shoulder region, clavicular area, arm and elbow joint, is performed in the interscalene groove. Although this groove is easy to locate, the local anaesthetic does not sufficiently anaesthetise the inferior trunk. Therefore, this approach is not recommended for surgery of the forearm or hand. The most common complications for this approach include hemidiaphragmatic paralysis and Horner's syndrome which are directly related to the volume of local anaesthetic used. ${ }^{2}$

The infraclavicular approach targets the cords of the BP and is indicated for surgery of the distal arm, elbow, forearm and hand. This approach is often used for the placement of indwelling catheters as the infraclavicular site provides stable positioning. Complications that can occur include chylothorax, which is a rare complication that occurs when performing left-sided blocks, and pneumothorax which occurs more frequently as compared to the supraclavicular approach.

The axillary brachial plexus block, is performed at the level of the terminal branches of the BP and may require multiple injections. Complications that may occur are specific to each terminal nerve. However, intravascular injection is more common in this approach compared to other approaches. ${ }^{2}$

\section{Indications}

The supraclavicular block is indicated for provision of an anaesthetic block for surgery or intra- and postoperative pain control for the entire upper limb including the shoulder, arm, elbow, forearm and the hand. ${ }^{2,3}$ Fractures, dislocations, abscesses as well as general surgery of the upper extremity for both adults and children, are examples of common indications for this block. ${ }^{2,3}$ Furthermore, it provides analgesia to the upper extremity for post-surgical and traumatic pain, complex regional pain syndrome, postamputation pain, vascular diseases and tumour-related pain. Relative and absolute contraindications must be considered prior to its utilisation and may include preexisting neuropathies or nerve injury and severe coagulopathies or anticoagulants. Other contraindications that may arise include infection at the site of injection, the inability to insert the needle at the injection site due to a splint or cast that may be present, or allergies to local anaesthetics. ${ }^{4}$

\section{Brachial plexus}

The BP is a network of nerves formed by the union of the ventral rami of the fifth to eighth cervical spinal nerves (C5-C8), as well as 
the first thoracic spinal nerve (T1). From proximal to distal, the BP consists of five roots ( $\mathrm{C} 5-\mathrm{T} 1)$ that will eventually unite into three trunks (superior, middle and inferior), six divisions (three anterior and three posterior), three cords (medial, lateral and posterior) and five terminal branches (axillary, musculocutaneous, radial, median and ulnar nerves). ${ }^{5}$

\section{Anatomical considerations}

The supraclavicular block is performed where the BP passes between the anterior and middle scalene muscles and follows the subclavian artery (SCA) into the arm between the clavicle and the first rib. The SCA lies anteromedial to the BP whereas both the latter two structures pass laterally and posterior to the anterior scalene muscle. The subclavian vein (SCV) accompanies the BP and SCA as it travels towards the root of the neck. It passes anterior to the anterior scalene before joining the internal jugular veins at the origin of the brachiocephalic veins. The middle scalene lies posterior to the BP and SCA and forms a very important landmark - the interscalene groove - together with the anterior scalene muscle. The first rib and pleura of the lungs can be found inferior to the BP and should be avoided when inserting the block needle (Figure 1). The superficial nerves innervate the proximal part of the arm, while the deeper nerves, which are closer to the first rib, innervate the distal part of the arm. $^{6}$

During the supraclavicular block, dermatomes $\mathrm{C} 5$ through $\mathrm{T} 1$ (i.e. the complete BP) are blocked, therefore providing anaesthesia for the entire upper extremity, including the shoulder region. Nerve branches given off from the roots of the BP provide motor innervation to selected muscles and structures. These nerves include the long thoracic nerve, which innervates the serratus anterior muscle; the dorsal scapular nerve that innervates both the levator scapulae and the rhomboid muscles; and a branch of the phrenic nerve, innervating the diaphragm. From the trunks of the BP, two nerves are given off, both of which provide motor innervation. These nerves are the suprascapular, which innervates the supra- and infraspinatus muscles, and the nerve to subclavius, which innervates the subclavius muscle.

Nerves given off from the cords of the BP have single or dual supply (motor and sensory innervation). The medial pectoral nerve provides motor innervation to the pectoralis minor and

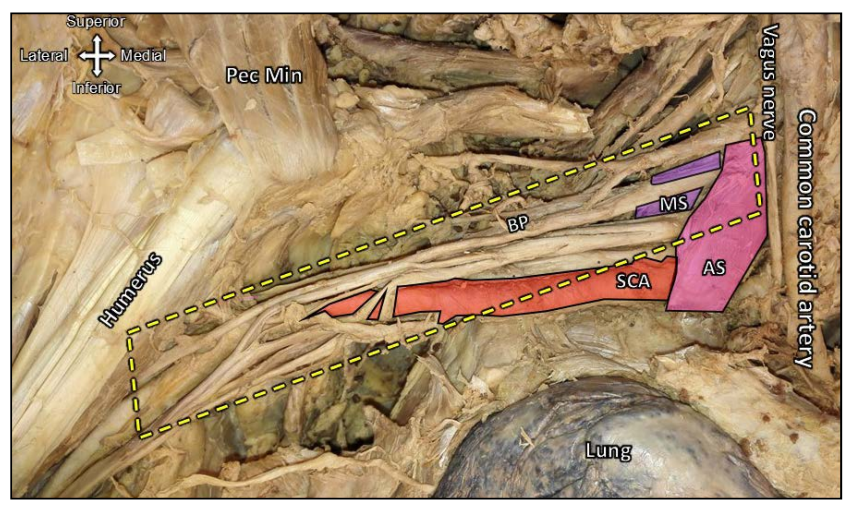

Figure 1. Pectoralis minor (Pec Min) reflected to reveal the course of the BP in the anterior neck and clavicular region. The BP and SCA can be seen passing between the anterior scalene (AS) and middle scalene (MS) muscles. The relationship of the structures to the lung is also clear. major muscles, whereas the lateral pectoral nerve provides motor innervation solely to the pectoralis major muscle. The medial cutaneous nerve of the arm provides sensory innervation to the skin above the medial side of the arm and the medial cutaneous nerve of the forearm provides sensory innervation to the skin above the medial side of the forearm as far distal as the wrist. The thoracodorsal nerve provides motor innervation to the latissimus dorsi muscle. The superior and inferior subscapular nerves collectively provide motor innervation to the subscapularis; the latter nerve also supplies the teres major muscle.

Lastly, all the terminal branches of the BP provide simultaneous motor and sensory supply. From the posterior cord, the axillary and radial nerves are located posterior to the axillary artery (AA). The former provides motor innervation to the deltoid and teres minor muscles, including sensory innervation to the skin above the deltoid and superior-posterior area of the arm. The radial nerve, which lies posterior to the AA, provides motor innervation to all muscles of the posterior arm and forearm (extensor compartment of arm and forearm), including sensory innervation to the skin above the posterior aspect of the arm and forearm, as well as the lateral two thirds of the dorsum of the hand and fingers (the area of the first web space). From the lateral cord, the musculocutaneous nerve pierces through the coracobrachialis muscle to provide motor innervation to all muscles of the anterior arm (flexor compartment of the arm) and sensory innervation to the skin above the lateral part of the forearm. The ulnar nerve is a branch of the medial cord and descends into the forearm to provide motor innervation to the intrinsic muscles of the hand, the flexor carpi ulnaris and the medial half of the flexor digitorium profundus muscles. The ulnar nerve also provides sensory innervation to the skin over the medial one and a half fingers of both the dorsum and palm of the hand. Finally, the median nerve forms from a contribution from both the medial and lateral cords. It descends in the arm lateral to the brachial artery and provides motor innervation of the anterior forearm (flexor compartment of the forearm) and sensory innervation to the skin above the lateral two-thirds of the palm and fingers. ${ }^{6}$

\section{Step-by-step procedure for ultrasound-guided supraclavicular BP block}

\section{Step 1: Patient position}

For this approach, the patient should be asked to lie in the semiFowler's position, i.e. in a supine position with the trunk raised between 15 and 45 degrees. The head should be turned to the contralateral side of where the block is to be performed with his/ her ipsilateral arm abducted and/or flexed at the elbow joint. The anaesthesiologist performing the block should be positioned at the head of the bed, with the patient, the block area and the ultrasound screen all in line, ensuring a clear view during the procedure (Figure 2). The probe should be held perpendicular to the skin.

\section{Step 2: Preparation}

While the patient is in a supine position, a sterile sheet should be placed over the thoracic area keeping only the area(s) of 


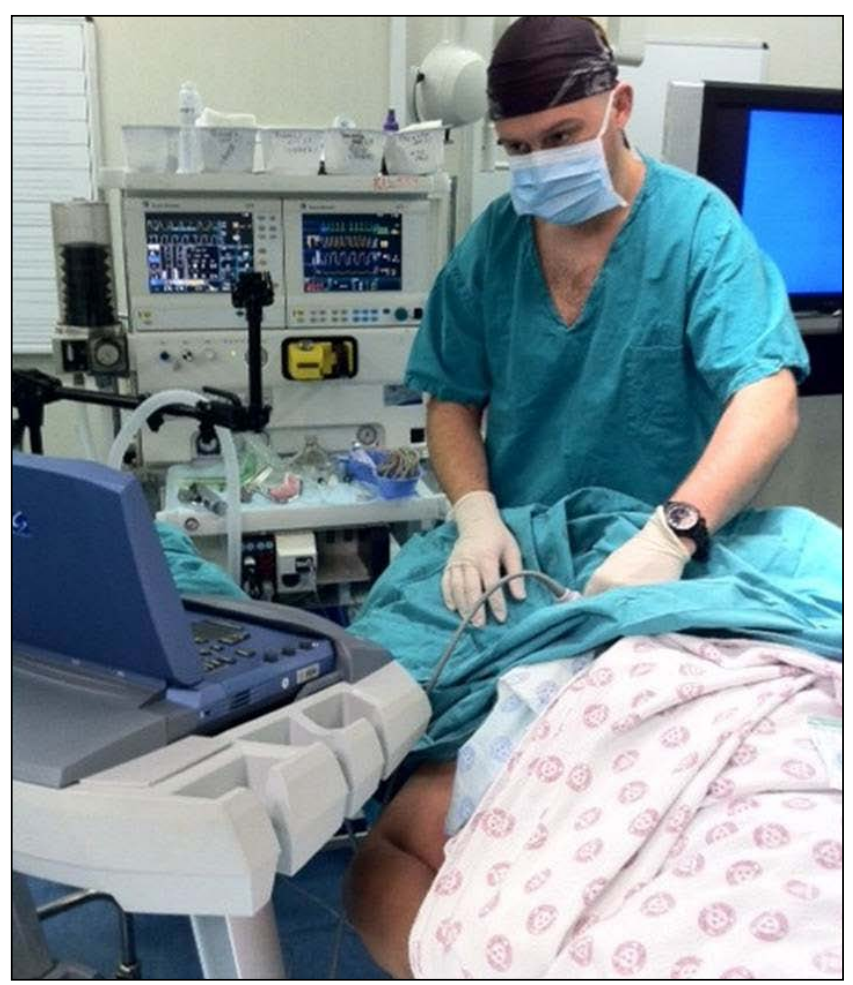

Figure 2. Patient lying in a supine position with the head of the bed elevated, and their head turned to the contralateral side of the block (semi-Fowlers position). This photo was included with the consent of DrMöhr.

interest - the supraclavicular fossa - exposed. A small amount of sterile ultrasound gel should be placed on the skin above the clavicle and posterior border of the sternocleidomastoid muscle, as well as on the ultrasonic transducer (probe) selected for the procedure. For ultrasound-guided BP blocks, a highfrequency linear probe is preferred. For this approach, a $21 \mathrm{G} \mathrm{x}$ $100 \mathrm{~mm}$ echogenic SonoPlex STIM needle is recommended and should be used to inject the anaesthetic solution.7 Strict aseptic techniques should be adhered to during the entire procedure.

\section{Step 3: Palpate and mark important landmarks}

Prior to starting the procedure, the entire clavicle should be palpated using the index and middle fingers of the nondominant hand and marked at the midpoint. This also applies to the borders of the sternocleidomastoid muscle, specifically the clavicular head of the sternocleidomastoid muscle and the interscalene groove (Figure 3). The interscalene groove or tissue space is situated between the anterior and middle scalene muscles at the level of the cricoid cartilage (vertebral level C6), ${ }^{7,8}$ The groove can be identified by rolling the palpating fingers from the posterior border of the sternocleidomastoid muscle posterolaterally over the belly of the anterior scalene muscle into the interscalene groove. The BP is deep to the supraclavicular fossa and posterior to the mid-clavicle.

\section{Step 4: Probe placement}

After palpating the clavicle and the clavicular head of the sternocleidomastoid muscle (if possible), the linear probe is placed in the supraclavicular fossa, superior and parallel to the midpoint of the clavicle, adjacent to the posterior border of the sternocleidomastoid muscle. The placement of the probe is in a parasagittal or coronal-oblique plane (Figure $4 \mathrm{~A}$ ) to obtain an optimal short-axis view of the SCA, first rib, pleura, lungs and the trunks/divisions of the BP. It may be necessary to rotate the probe (clockwise and counter clockwise) to obtain the ideal ultrasound anatomy imaging of these structures (Figure 4B). The probe is initially aimed inferiorly in the direction of the chest or mediastinum.

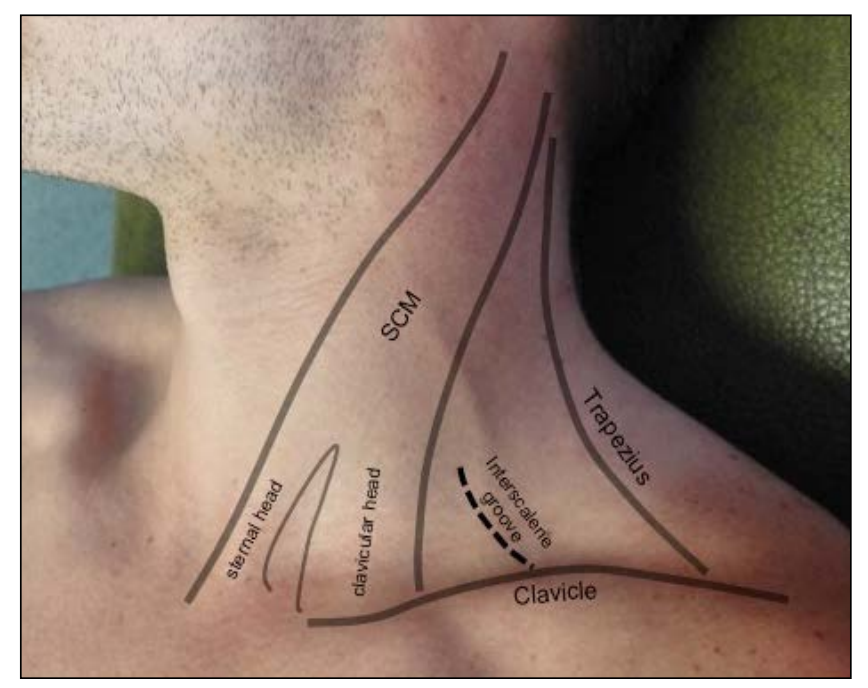

Figure 3. Photograph showing the posterior triangle of the neck. The clavicular head of the sternocleidomastoid (SCM) and clavicle form the main landmarks for palpating the interscalene groove between the anterior and middle scalene muscles.

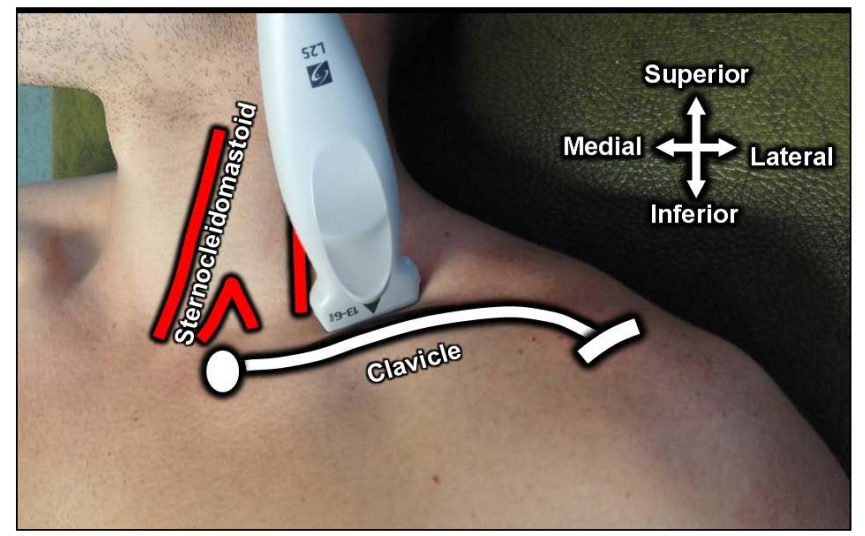

Figure 4A. Superior view of the probe placed in the supraclavicular fossa (posterior and approximately parallel to the clavicle).

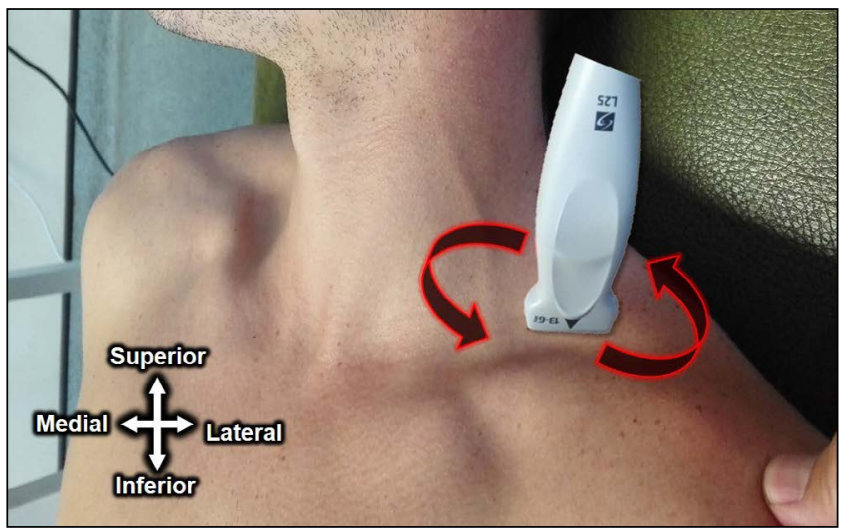

Figure 4B. Rotation of the probe for ideal sonoanatomy imaging. 


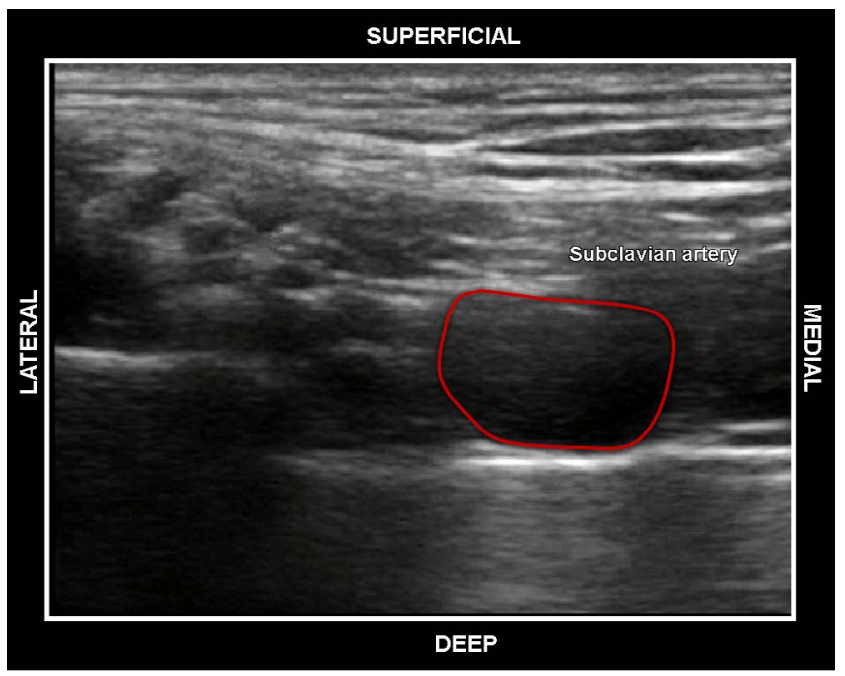

Figure 5. SCA presents as a hypoechoic structure on the ultrasound screen.

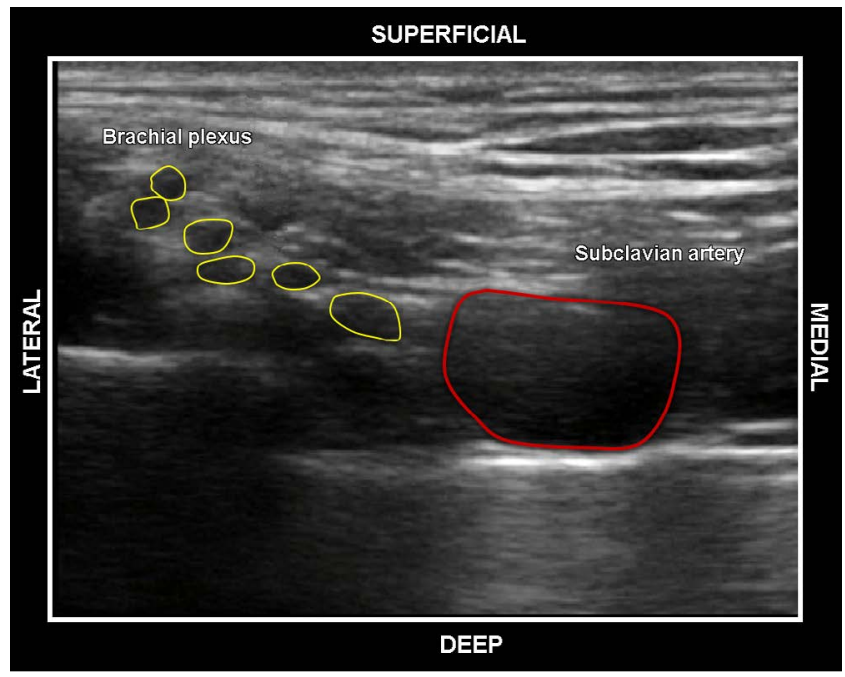

Figure 6. BP (grape-like appearance) found postero-lateral to the SCA.

On the ultrasound screen, one should expect to visualise the SCA (the key sonographic landmark) as a round, pulsating hypoechoic structure (Figure 5). The presence of the SCA can be confirmed through colour Doppler.

The BP has a grape-like appearance, with several hypoechoic bundles embedded in a hyperechoic supporting connective tissue surrounded by epineurium, found posterolateral to the $\mathrm{SCA}^{9}$ (Figure 6).

\section{Step 4: Summary of landmarks visualised on the ultrasound image}

The first rib can be identified as a hyperechoic structure at the bottom of the screen. Its reflective properties make the rib appear as a white horizontal/oblique line (Figure 7). Thereafter, the SCA can be identified as a hypoechoic ring, or a dark, round pulsating circle superficial to the rib. The pulsating artery is confirmed using the colour doppler function on the ultrasound machine, which will appear in red (due to the flow of blood towards the probe). The BP is found cephalad to the SCA. The middle scalene muscle can be identified posterolaterally and the

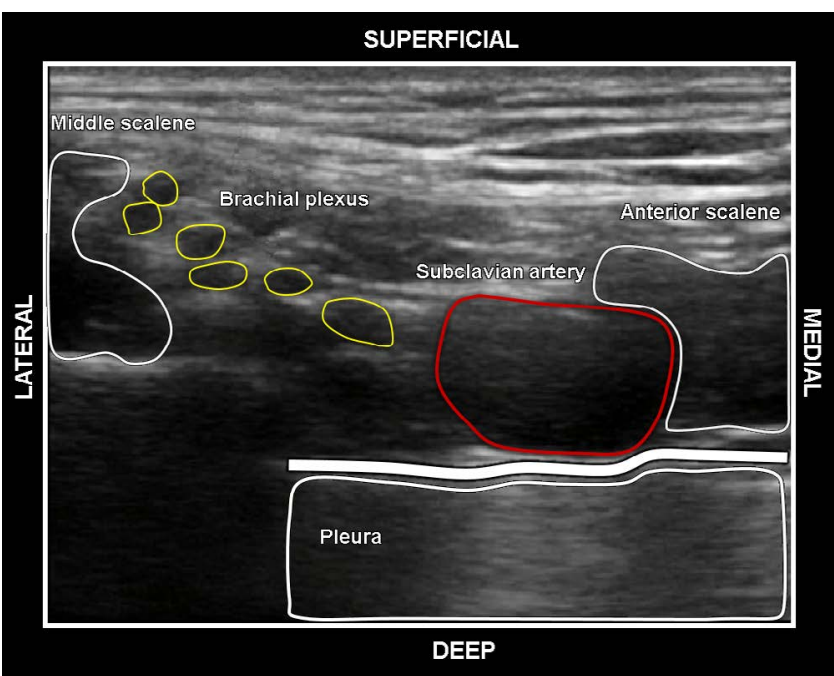

Figure 7. Ultrasound image showing the muscular- and vascular structures in the supraclavicular fossa.

anterior scalene anteromedially. Inferior to the first rib the lung can be viewed as a greyish mass, with the area below the level of the first rib referred to as the danger area ${ }^{9}$ (Figure 7). If the needle advances below this point there will be a high risk of penetrating the parietal pleura, resulting in a pneumothorax.

\section{Step 5: Needle insertion and course}

The needle should be inserted using the in-plane technique at the lateral border of the probe (Figure $8 \mathrm{~A}$ ). Once the skin has been pierced, the needle should be advanced in a lateral to medial direction - in the same plane as the ultrasound beam towards the BP located posterolateral to the SCA. The reason for advancing the needle in the same plane as the ultrasound probe is so that the entire length of the needle and the needle tip is visible as it is directed towards the BP. The needle should pierce the axillary sheath, which offers a small amount of resistance or the characteristic 'pop' sensation. The needle tip is then carefully directed as close to the BP as possible. The ideal location although dependent on the site of surgery and the intended extent of the block coverage - is at the junction of the BP and

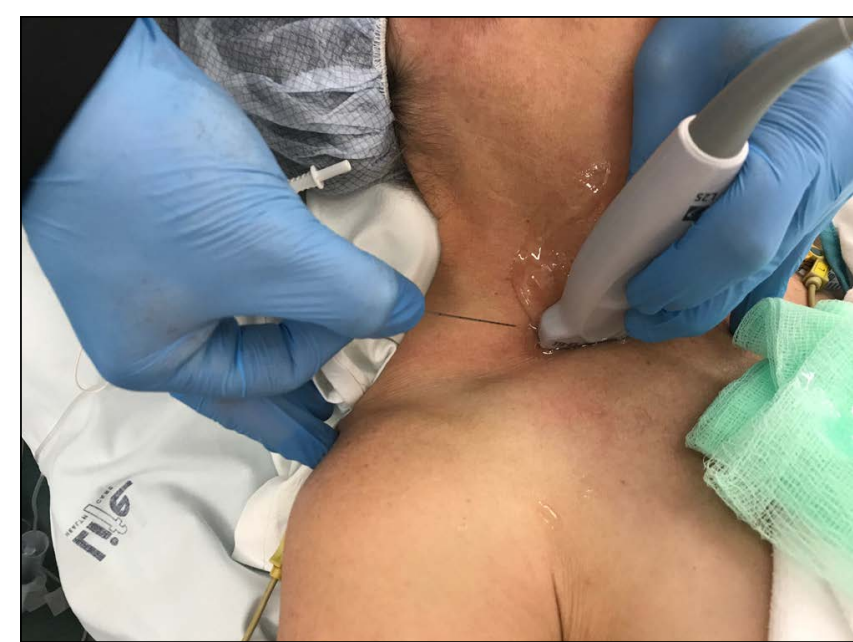

Figure $8 \mathrm{~A}$. In-plane needle insertion at the lateral border of the probe during an ultrasound-guided supraclavicular BP block. This photo was included with the consent of Dr Möhr. 

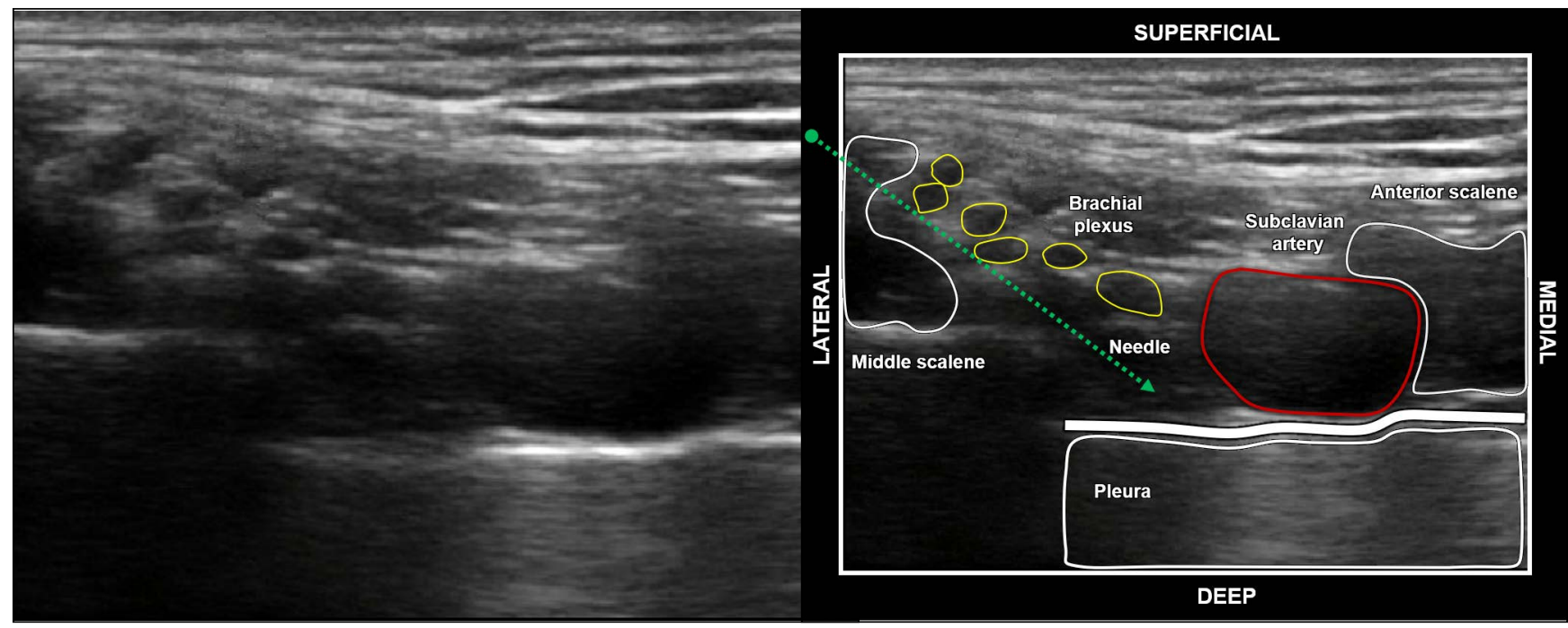

Figure 8B. Image on the left - ultrasound scan as it appears on the ultrasound screen during an ultrasound-guided supraclavicular BP block. Image on the right - In-plane needle insertion with the entire needle course visible on ultrasound screen during an ultrasound-guided supraclavicular BP block.

the posterolateral border of the SCA, superior to the first rib (Figure $8 \mathrm{~B}$ ). This area is referred to as the "corner pocket" or common injection site for this approach. ${ }^{10}$

\section{Step 6: Injecting local anaesthetic solution}

Once the tip of the needle has been confirmed, the local anaesthetic can be injected in the vicinity of the BP (Figure 9). Alternatively, the anaesthetic may be injected between the divisions of the plexus using the 'hydro-dissection' technique. ${ }^{9}$ Placing the local anaesthetic within the axillary sheath - the fascia that is continuous from the prevertebral layer of the cervical fascia and surrounds the components of the BP - will result in a faster onset and more extensive coverage. However, it may cause a higher risk for intraneural injection or injury. Deciding on the concentration of anaesthetic depends on whether the block is being performed for anaesthetic or analgesic procedures. In both cases the recommended volume will remain the same $(20 \mathrm{ml})$. However, the dilution of the anaesthetic agent will differ. For surgical procedures, $20 \mathrm{ml}$ of $0.5 \%$ of bupivacaine

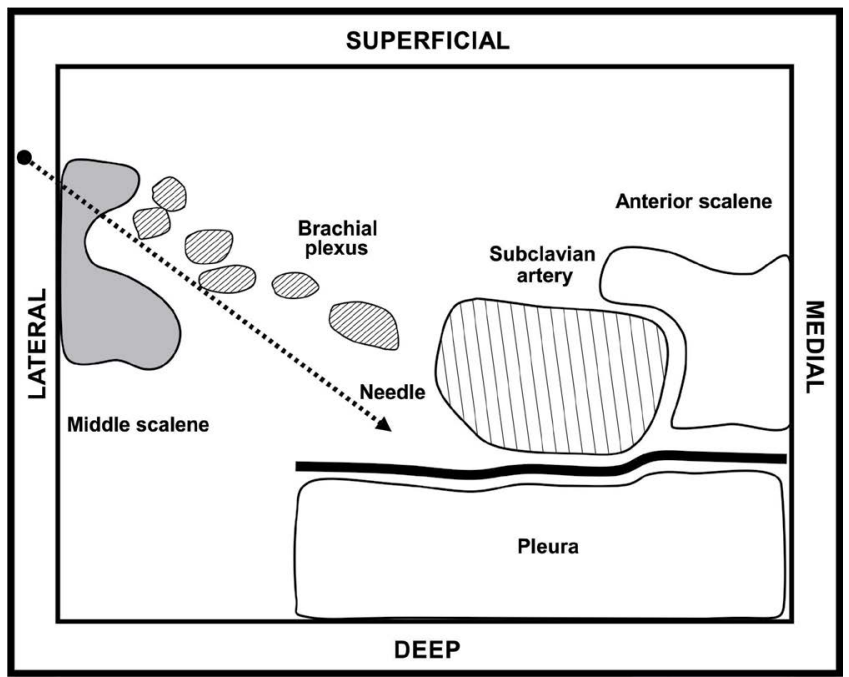

Figure 9. Line showing the course of the needle, whilst highlighting the important structures visible. (an intrinsic vasodilator) is recommended whereas, for analgesic blocks, $20 \mathrm{ml}$ of $0.25 \%$ or $0.125 \%$ of bupivacaine is recommended. To reduce the risk of systemic toxicity, it may be prudent to use a lower total dose of local anaesthetic or to consider the use of less cardiotoxic drugs such as ropivacaine or levobupivacaine. Ropivacaine and levobupivacaine may prolong the duration of action and slow systemic absorption due to its intrinsic vasoconstrictive properties. ${ }^{10}$ Additionally, adrenaline can be used as an adjuvant as it may reduce the absorption rate and therefore, potential systemic toxicity. The needle must be flushed with a local anaesthetic to prevent injection of air (which will create imaging artefacts).

\section{Step 7: Visualising the spread of the local anaesthetic solution}

Once the axillary sheath has been penetrated, a slight decrease in resistance or a 'click' or 'pop' can be felt. The anaesthetic solution within the syringe can now be injected slowly within the axillary sheath. As the local anaesthetic is being injected,

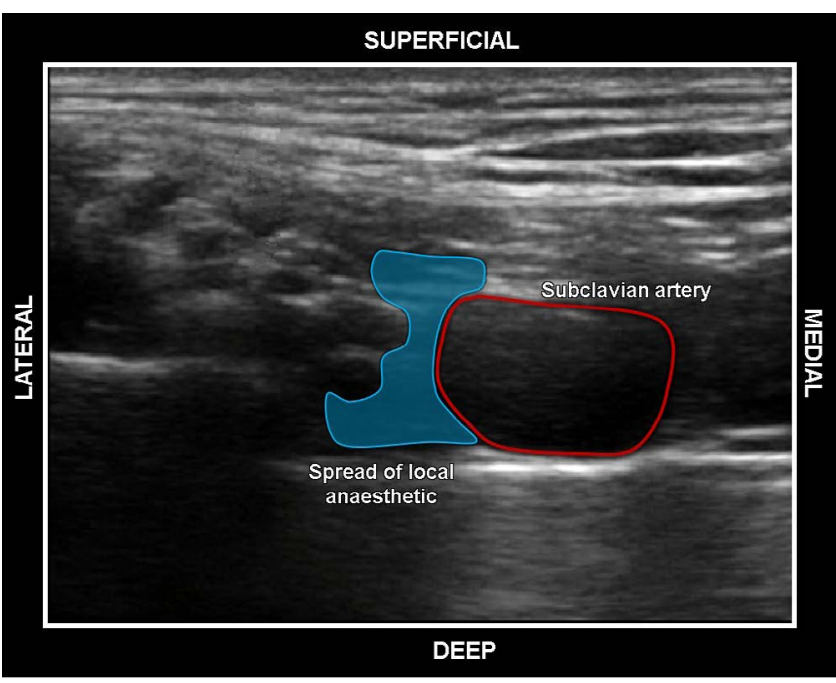

Figure 10. Spread of local anaesthetic solution (hypoechoic area high around the SCA) around the $\mathrm{BP}$, concealing it from view on the ultrasound machine. 
the spread of the solution around the BP should be observed on the screen. Injecting the anaesthetic solution into the axillary sheath will cause the nerve bundle to separate. Positioning the needle tip and subsequently injecting the local anaesthetic outside the axillary sheath will require higher volumes of local anaesthetic to be injected to ensure adequate anaesthesia. The local anaesthetic solution appears as a hypoechoic fluid and appears black on the ultrasound image (Figure 10).

The exact volume can then be judged, and the injection stopped once all the nerve structures have been bathed in local anaesthetic solution, thus avoiding the introduction of excessive and unnecessary amounts of local anaesthetic.

\section{Advantages of ultrasound-guided supraclavicular BP blocks}

In the literature, various studies have been done to compare the relative blocks for upper limb procedures, most of which conclude that the supraclavicular block has the highest success rates and greater anaesthetic spread. ${ }^{11}$ This is mainly because the anaesthetic solution is delivered at the level of the trunks/ divisions of the BP. This is a compact part of the BP which ensures rapid onset and since the needle typically has a maximum depth of $1.5 \mathrm{~cm}$ to be inserted above the clavicle, there are fewer incidences of pneumothorax. ${ }^{11}$ Success rates for the supraclavicular BP have been reported to be $85 \%$ and above. ${ }^{12}$

Despite the benefit of ultrasound guidance, complications may still occur. Additional studies have been done to evaluate the prevalence of complications that may occur with and without the aid of ultrasound guidance. The reported incidence of pneumothorax without ultrasound guidance ranges between $0.5-6 \%$ whereas with the aid of ultrasound guidance the incidence is $1 \% .^{13,14,15}$ Other complications that may occur include injury to the recurrent laryngeal nerve resulting in a hoarse voice (a reported incidence of 1\%), phrenic nerve injury resulting in hemidiaphragmatic paresis (a reported incidence of $33 \%$ ), and vascular puncture (a reported incidence of $0.4 \%$ ). ${ }^{13,14,15}$ Horner's syndrome has also been described and results from paralysis of the ipsilateral sympathetic cervical chain caused by drugs, surgery or local compression (a reported incidence of $1 \%)^{13,14,15}$ The latter can be caused by the injection of large volumes of local anaesthetic or the atypical proximal migration of the solution above the clavicle toward the interscalene area. ${ }^{15}$ Horner's syndrome is classified by a triad of symptoms including miosis, a condition in which there is excessive constriction of the pupil; ptosis or drooping of the upper eyelid; and anhidrosis - the absence of perspiration or failure of the sweat glands. ${ }^{16}$ The rate of complications is also related to the experience of the anaesthetist. ${ }^{16}$ Thus, good technique, regular practice and a sound knowledge of human anatomy are vital in performing the supraclavicular nerve block whether under ultrasound guidance or not.

\section{Conclusion}

Using ultrasound during invasive procedures is of growing importance in the medical field, assisting doctors and surgeons with many clinical procedures. Ultrasound guidance for the supraclavicular BP block is clinically beneficial for nerve localisation as it reduces the number of needle attempts and may also decrease the number of failed blocks. If anatomical structures can be visualised in real-time, then it is possible for anaesthesiologists to define the correct needle insertion site, the depth at which the needle needs to be inserted, identify the optimal site of injection, as well as avoid complications such as neurovascular injuries or pleural puncture. Having good anatomical knowledge of the supraclavicular fossa and structures found deep to the skin is important, as ultrasound guidance has proven to increase the confidence of doctors performing regional nerve blocks, decrease complications and failed block attempts, and increase the success rates of not just supraclavicular brachial plexus blocks, but most regional nerve blocks. ${ }^{17,18}$

This description of our approach to ultrasound-guided supraclavicular BP block serves as a comprehensive step-bystep guide to performing this block safely and successfully. It also aims to provide the reader with the background behind the technique and to describe the anatomy of the region where the block is performed.

\section{ORCID}

\section{S Govender (i) https://orcid.org/0000-0001-6738-9071 A van Schoor (D) https://orcid.org/0000-0001-9813-0441 Z Tshabalala (D) https://orcid.org/0000-0001-6889-5283}

\section{References}

1. Bharti N, Bhardawaj N, Wig J. Comparison of ultrasound-guided supraclavicular, infraclavicular and below-C6 interscalene brachial plexus block for upper limb surgery: a randomised, observer-blinded study. Anaesth Intensive Care. 2015:43(4):468-72.

2. Wankhade H, Chauhan VP, Parikh R, Saxena AK. Ultrasound-guided supraclavicular brachial plexus block for the upper limb fracture or dislocation reduction in emergency in emergency department. IRPMS. 2017;2(5):16-21.

3. Möhr D. When will the sonar ping reach our shores? SA J Anaesth Analg. 2010;16(3):4.

4. The New York School of Regional Anaesthesia. Available form: www.nysora.com Cited 20 February 2011. Accessed 12 April 2016.

5. Pham Dang C, Gunst JP, Gouin F, et al. A novel supraclavicular approach to brachial plexus block. Anaesth Analg. 1997;85(1):111-6.

6. Moore KL, Dalley AF, Agur AMR. Clinically Oriented Anatomy. 6thed. Philadelphia: Lippincott Williams \& Wilkens; 2010.

7. Leonard B, Papper EM. A comparison of supraclavicular and axillary techniques for brachial plexus blocks. Anaesthesiology. 1961;22:226-9.

8. Jung Kim H, Hyun Park S, Young Shin H, Choi YS. Brachial plexus injury as a complication after nerve block or vessel puncture. Korean J Pain. 2014;27(3):210-8

9. Demondion $X$, Herbinet $P$, Boutry $N$, et al. Sonographic mapping of the normal brachial plexus. AJNR am J Neuroradial. 2003;24(7):1303-9.

10. Linsey E, Picard J, Weiberg G. Local anaesthetic systemic toxicity. BJA Education. 2015;15(3):136-42.

11. Hanumanthaiah D, Vaidiyanathan $S$, Garstka M, Szucs S, lohom G. Ultrasound guided supraclavicular block. J Med Ultrasound. 2015;15(3):2224-9.

12. Patil S, Koshire A. Comparison of success rate of classical supraclavicular brachial plexus block with and without nerve stimulator. MVP Journal of Medical Sciences. 2017:4:6.

13. Govender S, van Schoor A, Möhr D. A critical analysis of macro- and sonographic anatomy of the brachial plexus. (Unpublished).

14. Perlas A, Lobo G, Lo N, et al. Ultrasound-guided supraclavicular block: outcome of 510 consecutive cases. Regional Anesthesia and Pain Medicine. 2009;34(2):171-6.

15. Gauss A, Tugtekin I, Georgieff M, et al. Incidence of clinically symptomatic pneumothorax in ultrasound-guided infraclavicular and supraclavicular brachial plexus block. Anaesthesia. 2014;69(4):327-36.

16. Helayel PE, Conceicao DB, Oliveria Filho GR. Ultrasound-guided nerve block. Braz J Anaesthesiol. 2007;57(1):106-123.

17. Bhaskaran K, Raju SV, Sriranjini, Thyagarajan N, Jalakandan. Ultrasound-guided supraclavicular brachial plexus block. Apollo Medicine. 2008;5:26-8. 